\title{
On the analysis of WiFi communication and WiMAX network entry over single radios
}

\author{
Sven Wiethölter*, Marc Emmelmann ${ }^{\dagger}$, Yerong Chen*, and Adam Wolisz* \\ *Telecommunication Networks Group (TKN), Technical University Berlin, Germany \\ ${ }^{\dagger}$ Fraunhofer Institute for Open Communication Systems (FOKUS), Berlin, Germany \\ Email: wiethoel@tkn.tu-berlin.de
}

\begin{abstract}
Future wireless mobile devices will have to support a variety of heterogeneous access technologies while having a limited number of transceiver chains. One of the most challenging tasks remains how to support wireless access in one of the technologies, while preparing a "smooth transition" to another, especially to technologies with a lengthy network entry such as WiMAX. This work proposes an efficient way to organize a WiMAX network entry process while continuing a communication session with stringent QoS requirements via WLAN. We advocate the prioritization of the strict WiMAX timing over the contention-based WLAN channel access. Our results show the applicability of this approach over a wide range of mobile WiMAX parameters and identify bounds on the WiMAX downlink load for which the WiFi communication does not suffer quality distortions.
\end{abstract}

\section{INTRODUCTION}

The diversity of heterogeneous wireless access technologies used by individual terminals (e.g., laptops, smart phones, tablets) has been growing continuously over the past few years. Bluetooth, WLAN, and some wide area wireless technologies count nowadays as a standard mix for these devices. An extensive set of technologies would include Bluetooth, IEEE802.11a/b/g/n, WiMAX, GSM, GPRS, Edge, HSPA, LTE, and even FM and DVB(-T). This wide deployment has several implications. On the one hand, users depend increasingly on the (preferably high speed) connectivity for everyday activities and express clear dissatisfaction if this connectivity is not available. On the other hand, the growth of wireless traffic leads to an increase of interference and to throughput bottlenecks.

Dynamic decisions about the access technology to be used (e.g. pushing the users from a cellular network to a WLAN) seem to be one of the promising approaches for both mitigating interference and overcoming the throughput bottlenecks. While quite a lot of research is devoted to a proper selection of the access technologies [1], it is frequently taking for granted that the switching itself, i.e., an enforced handover from one technology to another, is somehow easily done.

Switching from one technology to another while supporting a continuous communication is, however, by far not trivial. The process of deciding about the availability of another technology, selecting a proper access point, and finally completing the association procedure can take a significant amount of time. Thus, it is hardly recommendable to enforce a hard handover, i.e., breaking the ongoing communication association before assuring that another, better connectivity is really available.

Realizing a soft handover with service continuity is restricted, however, by the applied communication hardware. Even if multiple network interface cards (NICs), each supporting one of the technologies, were available, their parallel usage would be avoided in order to economize energy. In reality nowadays, there is a strong trend to limit the number of separate NICs within a single device due to space and cost issues. As a result, multi-standard NICs become a solution of choice. Such multi-mode radios can support multiple technologiesbut at a given time only access to one wireless technology over a specific transceiver chain is possible.

This work deals with the problem how to enable a usage of heterogeneous links over such multi-standard NICs while meeting QoS constraints of an on-going transmissionincluding constraints imposed by real-time voice connections. While following a more general concept, we present in detail a solution compatible to the family of IEEE 802.11 and 802.16 standards. We identify the fundamental limits of this novel approach by considering the timing issues for both WLAN communication and the lengthy WiMAX network entry [2].

The rest of this paper is structured as follows. Section II summarizes the relevant related work. Section III specifies our system under consideration and gives the problem formulation, while our approach together with its analysis for WLAN and WiMAX is highlighted in Section IV. Next, Section V presents and discusses results regarding the general feasibility of our approach. Finally, Section VI concludes our work.

\section{RELATED WORK}

In order to enable heterogeneous wireless access anywhere and anytime by just a limited number of NICs within a device, a hybrid approach is common today: multi-mode, reconfigurable radios are able to do MAC as well as some PHY (base-band) processing in pure software, but still apply transceiver chains with specific analog parts (amplifier, filter) that are specifically designed and adopted to their purposes and frequency bands (e.g. joint WLAN/WiMAX and 2G/UMTS/LTE transceiver chains) [3]. Vendors like Infineon or Intel [4], [5], for example, have been developing dual-mode WLAN/WiMAX transceiver chips that have been incorporated into todays' mobile devices.

A trivial solution may simply switch the transceiver chain from one access technology to the other, imposing a hard 
vertical handover. Choi et al. [6] propose a more sophisticated heterogeneous handover scheme, however for multiple NICs, in which it is possible to have only a single NIC active during each time instance to account for the limited battery power and radio resources. The authors utilize the mutual silence periods of ON/OFF traffic, such as VoIP, for the execution of handovers. Both, hard vertical handovers as well as Choi's scheme, may be promising approaches for technologies with quick Layer 2 associations, but may suffer in case of handovers to technologies such as WiMAX, which are known to have a very lengthy network entry process [2] thus exceeding the length of mutual silence periods by magnitudes.

Instead, we consider to alternate the ongoing communication in the first radio access technology (RAT) with the link setup in the second RAT on properly chosen time scales.

The only work addressing simultaneous, QoS-constrained transmissions and neighbor discovery over the same NIC on small time scales has been presented in the context of homogeneous WLANs [7]. This approach, denoted as opportunistic scanning, utilizes IEEE 802.11 Power Save mode (PS) to shortly pause the on-going communication and scan other WLAN channels passively with intervals in the area of just some milliseconds. The theoretical limits for finding another WLAN cell with a given probability have been presented in [7], the follow-up work [8] shows the sturdiness of this approach even with background traffic; a patent application is pending for this homogeneous WLAN approach [9].

Still, it remains an open point how to realize a similar approach in a standard-compliant way for neighbor discovery or even handovers to a second, heterogeneous technology in which timing constraints and requirements regarding medium access differ tremendously from 802.11.

\section{System AND PROBlem Formulation}

As shown in Fig. 1, we consider a user with a portable device like a notebook which is associated with an IEEE 802.11 WLAN access point (AP) and which is within the coverage of an IEEE 802.16e base station (BS) additionally. User's device is equipped with several multi-mode radios, whereby WLAN and WiMAX share the same dual-mode transceiver. This WLAN/WiMAX transceiver chain is able to switch among the RATs within insignificant time of a single clock cycle [10].

We focus on traffic from a user with nomadic mobility, i.e., he remains stationary during his communication sessions, as it is expected that more than 80 percent of the mobile data traffic will appear indoor in office or home scenarios, only [11].

For WLAN, we focus on IEEE 802.11g ERP OFDM with data rates ranging from 6 to 54 Mbps. For WiMAX, we consider the TDD mode and the parameters of the mobile profiles specified by the WiMAX Forum [12]. QPSK-1/2 is applied as Modulation and Coding Scheme (MCS) for DL-/UL-MAP, and DL/UL channel descriptor (DCD, UCD) messages.

Via the WLAN access cell, the user has an active VoIP communication session (ITU-T's G.711 voice codec with $20 \mathrm{~ms}$ packetization). Thus, the joint WLAN/WiMAX transceiver is

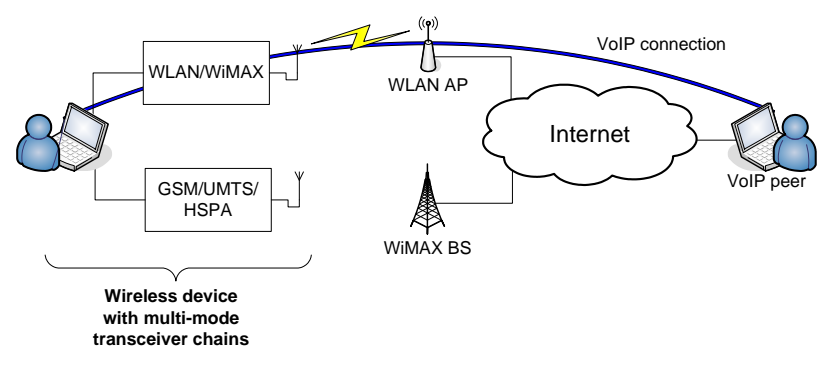

Fig. 1. Network Scenario

blocked, such that no simultaneous access to WiMAX may be possible. The VoIP call has the usual hard QoS constraints (maximum packet loss of 1 to 3 percent and a maximum tolerable one-way end-to-end delay of $150 \mathrm{~ms}$, cf. [13]).

There exist manifold reasons for lost or delayed VoIP packets. They may stem from the IEEE 802.11 access cell or the wired part, e.g., a DSL-provider, the Internet side or any combination of the involved entities. Even the wired part can significantly contribute to variations in the VoIP QoS. Several measurements over backbones paths have shown a temporal diverse behavior between different paths, whereby some of the paths have even periodic patterns with respect to temporal higher delays [14].

As a basis of this work, we assume that the end user with the wireless device perceives some variations in the VoIP QoS due to jittering delay in the wired part. Although they do not bring the overall QoS below the acceptable level immediately, the user has perceived a slight degradation. To circumvent a potential stronger impairment, a handover is imminent, although the wireless device itself perceives a good and lowloaded WLAN channel.

For this scenario, we consider a solution that alternates the ongoing VoIP over WLAN communication with the network entry process in WiMAX, which is the basis for the establishment of another path to the VoIP peer. In order to allow a fast setup of the alternative path, the WiMAX network entry process has to be conducted as quickly as possible. Thereby, the fundamental question appears how to still support the VoIP communication over the WLAN path additionally in a standard-compliant way.

\section{Heterogeneous OpPortunistic ApProAch}

\section{A. Principle}

Following the spirit of [7], [8], we pause WLAN by means of the power save mode and switch to WiMAX in the gaps (Fig. 2). Let us analyze the "quanta" in which the WiMAX entry process has to proceed. The first step consists of finding the WiMAX downlink channel and to adapt to the strict timing of the WiMAX frames afterwards. This is followed by the steps of obtaining DL/UL parameters, initial ranging, capability negotiation, authorization and key exchange, and network registration [2]. Lastly, the establishment of the IP connectivity and the service flow finally prepares WiMAX for the VoIP transport. 


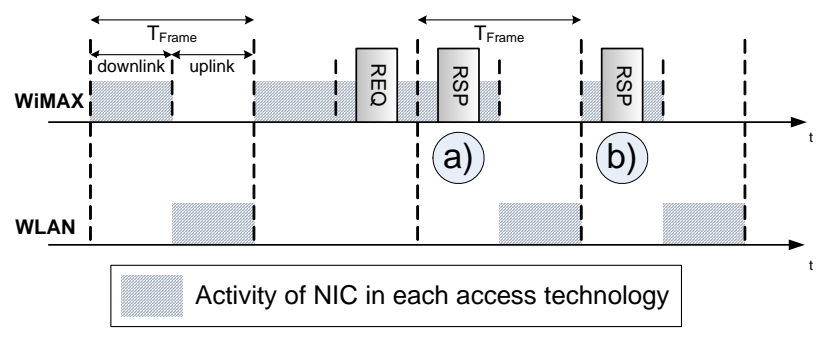

Fig. 2. WLAN-WiMAX alternation principle

In order to allow for a fast setup of the second path via WiMAX, we have to give strict timing priority to WiMAX in order to keep the duration of the network entry process small. Nevertheless, for other applications different from this work, one may further tradeoff the priority of WiMAX timing and WLAN channel access. In case of strongly fluctuating WLAN channels, for example, it may be important to give more channel access time to WLAN (i.e., prioritize the transport of VoIP data) and postpone subsequent steps of the WiMAX network entry process (in the valid range of the IEEE 802.16 standard) instead. Since this work limits us to keep the duration for the WiMAX network entry process as small as possible, we prioritize the WiMAX timing, i.e., the Mobile Station (MS) has to be present during the downlink (DL) part for all WiMAX frames. In case there is no pending action for the uplink (UL), MS switches to WLAN and returns back for the start of the next WiMAX frame.

Beside the neighbor discovery, all further steps of the network entry process for WiMAX [2] are based on request (REQ) / response (RSP) two-way handshakes, where MS issues the REQ and waits for the RSP of the Base Station (BS). For each of these steps, MS has to spend the complete frame plus the following DL subframe in WiMAX mode (in order to send out REQ and potentially receive RSP, if transmitted by BS immediately in the subsequent DL subframe, Fig. 2 case a)). If RSP will be sent later in one of the following frames, it will be received by MS anyway since it always spends the DL-part of the frame within WiMAX (Fig. 2 case b) .

The timing priority of WiMAX may lead to UL phases, in which MS cannot switch to WLAN because of pending actions. In these cases, WLAN access is delayed to the next WiMAX UL subframe. If a VoIP packet is awaiting a transmission in WLAN, this imposes a marginal additional delay of another WiMAX frame but ensures timely network entry in WiMAX.

\section{B. IEEE 802.16e Timing Issues}

The duration of the WiMAX DL subframe limits the available time for VoIP transmissions in WLAN and vice versa. The analysis takes into account the maximum duration of communication patterns in each technology, such that MS can be still present for WiMAX DL subframes and can transmit VoIP without any quality distortions in WLAN. For this, we assume as a starting point an idle channel in WLAN and no other active MS in WiMAX. Later in Sec. V-C we
TABLE I

PARAMETERS ACCORDING TO IEEE 802.16E AND WiMAX FORUM

\begin{tabular}{|c|c|c|}
\hline $\begin{array}{l}\text { frame duration }[\mathrm{ms}] \\
\text { bandwidth }[\mathrm{MHz}] \\
\text { cyclic prefix ratio }\end{array}$ & $\begin{array}{c}T_{\text {frame }} \\
\text { BW } \\
G\end{array}$ & $\begin{array}{c}5 \\
3.5,5,7,8.75,10 \\
1 / 8\end{array}$ \\
\hline FFT size & $N_{\text {FFT }}$ & $\begin{array}{c}512(3.5,5 \mathrm{MHz}) \\
1024 \text { else }\end{array}$ \\
\hline sampling factor & $n$ & $\begin{array}{c}28 / 25(5,10 \mathrm{MHz}) \\
8 / 7 \text { else }\end{array}$ \\
\hline \#PUSC subchannels & $N_{\text {DL-PUSC }}$ & $\begin{array}{l}15 \text { (512 FFT), } \\
30(1024 \text { FFT })\end{array}$ \\
\hline \#FUSC subchannels & $N_{\text {DL-FUSC }}$ & $\begin{array}{c}\text { (512 FFT), } \\
16 \text { (1024 FFT) }\end{array}$ \\
\hline $\begin{array}{l}\text { code rate } \\
\text { modulation level }\end{array}$ & $\begin{array}{c}c \\
m\end{array}$ & $\begin{array}{c}1 / 2,2 / 3,3 / 4 \\
2,4,6\end{array}$ \\
\hline modulation & & $\begin{array}{c}\text { QPSK, 16QAM \& } \\
\text { 64QAM }\end{array}$ \\
\hline \#Symbols & $\begin{array}{c}S_{\text {preamble }} \\
S_{\mathrm{FCH}, \text { DL-MAP }}\end{array}$ & 1 \\
\hline $\begin{array}{l}\text { DL burst \#1 [Byte] } \\
\text { DL burst \#2 [Byte] }\end{array}$ & $\begin{array}{l}L_{\text {burst }_{1}} \\
L_{\text {burst }_{2}}\end{array}$ & $\begin{array}{l}301 \\
163\end{array}$ \\
\hline
\end{tabular}

consider results for various DL loads in WiMAX, for the load dependency in WLAN the reader is referred to [8].

1) WiMAX: The duration of the DL part takes its maximum for the network entry process if DL/-UL-MAP, UCD, DCD (within DL-burst \#1) and (the largest) RSP message (DLburst \#2) are transmitted together in one DL subframe. Eq. 1 gives the duration of the DL subframe:

$$
\begin{aligned}
t_{\text {WiMAX-DL }}=t_{\text {symbol }}\{ & S_{\text {FCH, DL-MAP }}+S_{\text {preamble }} \\
& \left.+S_{\text {DL-PUSC }}+S_{\text {DL-FUSC }}\right\},
\end{aligned}
$$

where the number of symbols $S_{\text {DL-PUSC/FUSC }}$, the number of occupied slots $N_{\text {slot }}$, and the symbol duration $t_{\text {symbol }}$ are specified below:

$$
\begin{gathered}
\left.S_{\text {DL-PUSC }}=2\left\lceil\frac{N_{\text {slot }}}{N_{\text {DL-PUSC }}}\right\rceil \text { [symbols }\right], \\
\left.S_{\text {DL-FUSC }}=\left\lceil\frac{N_{\text {slot }}}{N_{\text {DL-FUSC }}}\right\rceil \text { [symbols }\right], \\
N_{\text {slot }}=\left\lceil\frac{L_{\text {burst }}[\text { Byte }] \cdot 8[\text { bit/Byte }]}{c \cdot m[\text { bit/data-sc }] \cdot 48[\text { data-sc/slot }]}\right], \\
t_{\text {symbol }}=(1+G) \frac{N_{\mathrm{FFT}}}{n \cdot \mathrm{BW}} .
\end{gathered}
$$

Table I summarizes selected parameters and their values according to IEEE 802.16e OFDMA [15] and the mobile profiles from the specification of the WiMAX Forum [12].

\section{IEEE 802.11 Timing Issues}

The maximum duration of VoIP transmissions in WLAN occurs, when AP and station (STA) apply the most robust data rate. Depending on the situation whether packets are awaiting their transmission in UL as well as DL or in one of the directions only, the power save (PS) signaling and its duration changes. 


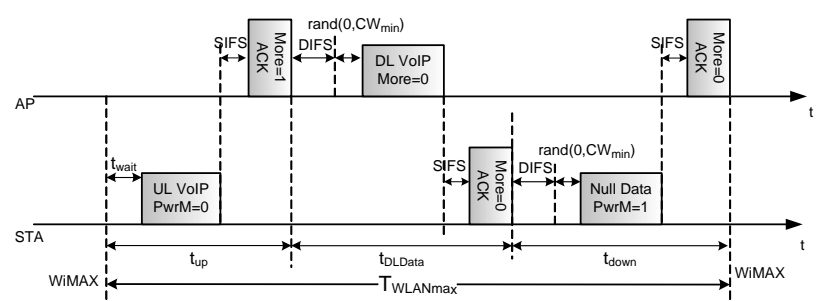

Fig. 3. WLAN PS signaling with up- and downlink transmission

TABLE II

$t_{\mathrm{WLAN}_{\max }}(\mathrm{ms})$ FOR IEEE $802.11 \mathrm{G}$ ERP OFDM

\begin{tabular}{|c||c|c|c|c|}
\hline \multicolumn{1}{|c||}{ (Mbit/s) } & Null data & in DL & in UL & in UL \& DL each \\
\hline \hline $\mathbf{5 4}$ & 1.230 & 1.386 & 1.258 & 1.414 \\
$\mathbf{6}$ & 1.350 & 1.806 & 1.618 & 2.074 \\
\hline
\end{tabular}

Fig. 3 shows the worst case, which consists of the wakeup, the exchange of one VoIP packet in UL and DL, and finally the sleep signaling. For this, Eq. 2 specifies the maximum active duration in WLAN:

$$
t_{\mathrm{WLAN} \text { max }}=t_{\mathrm{up}}+t_{\mathrm{DLdata}}+t_{\mathrm{down}},
$$

where:

$$
\begin{aligned}
t_{\mathrm{up}}= & t_{\mathrm{wait}}+t_{\mathrm{SIFS}}+t_{\mathrm{ACK}}+ \begin{cases}t_{\mathrm{VoIP}} & \text { pkt in UL, } \\
t_{\mathrm{Null}} & \text { else, }\end{cases} \\
t_{\mathrm{DLdata}}= & \begin{cases}0 & \text { no pkt in DL, } \\
t_{\mathrm{DIFS}}+t_{\mathrm{VoIP}}+t_{\mathrm{SIFS}}+t_{\mathrm{ACK}} & \text { else, }\end{cases} \\
& t_{\mathrm{down}}=t_{\mathrm{DIFS}}+t_{\mathrm{Null}}+t_{\mathrm{SIFS}}+t_{\mathrm{ACK}} .
\end{aligned}
$$

Table II gives the maximum active communication duration for IEEE 802.11g ERP OFDM (parameters as in [7], [16]), for the cases of no traffic, a packet in each direction only, and for both up- and downlink. For the cases with present VoIP traffic, the highest values for the most robust MCS with 6 Mbps have been selected as thresholds (highlighted in grey).

\section{Discussion of RESUlts}

\section{A. WiMAX Neighbor Discovery}

The first step of the heterogeneous opportunistic approach tackles the neighbor discovery of a WiMAX network. Hereby, we shortly derive constraints for the selection of the scanning interval similar to the work in [7].

On the one hand, the scanning duration will be maximized in order to speed up the WiMAX discovery process, i.e., to minimize the number of required scanning attempts. On the other hand, we propose to stay below the packet intergeneration time for the considered VoIP traffic, which is in this case $20 \mathrm{~ms}$, in order to not induce large additional delays. Moreover, the scan interval must not be equal to one or to multiples of the WiMAX frame sizes, since then the periodicity leads to problems in finding the other network. In [7] it is described that intervals with prime numbers fulfill

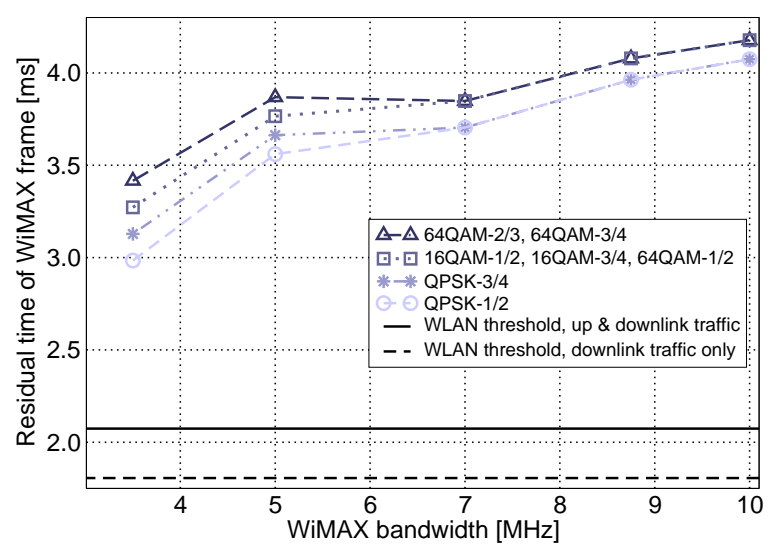

Fig. 4. Available residual time of WiMAX frame

this last requirement. Overall, this leads to an optimal scanning interval size of $19 \mathrm{~ms}$. With the choice of this interval value, a WiMAX network in a specific frequency band will be found in just one scanning interval if the WiMAX frame size ( $\left.T_{\text {WiMAX }}\right)$ is equal or smaller than $12.5 \mathrm{~ms}$, which is the case for mobile WiMAX with of $5 \mathrm{~ms}$ frames. With $T_{\text {Wimax }}$ of $20 \mathrm{~ms}$, Equation 4 in [7] holds and results in a maximum of 2,3 , and 4 scanning attempts with 5 percent probability each, while just a single attempt is required in 85 percent of the cases.

Compared to the task of finding another WLAN AP ([7]), the number of required scanning attempts for WiMAX is very low and can be seen as an uncritical part of the network entry process.

\section{B. Single MS: Feasible Parameter Space for Mobile WiMAX}

Once the neighbor discovery has been completed, all further steps of our approach require the MS to stay for the DL subframes within the WiMAX cell (cf. Section IV-A).

Now, when the MS stays in WiMAX for the duration of the DL subframe, a residual duration occurs, which is analyzed in the following. Since we assume that this residual time span is used for WLAN communication, $t_{\text {residual }}$ has to be greater than the WLAN thresholds defined in Section IV-C.

$$
t_{\text {residual }}=T_{\text {frame }}-t_{\text {WiMAX-DL }_{\text {f }}}>T_{\mathrm{WLAN}_{\max }}
$$

The residual time values were calculated for all combinations of channel bandwidths and MCSs (for DL-burst \#2) listed in Table I. Fig. 4 shows the results for the case of no other DL-load in WiMAX: the residual time of the WiMAX frame stays far above the WLAN thresholds for all parameter combinations. Thus, our approach is feasible if no other traffic is present.

\section{Multi-MS Case: Load-Dependency}

The last part deals with the influence of background traffic in the WiMAX DL subframe, i.e., BS serves also other MSs. This further reduces the time span that is utilized to switch to WLAN. We identify the performance limits for this duration as a function of present traffic in the WiMAX DL subframe. 


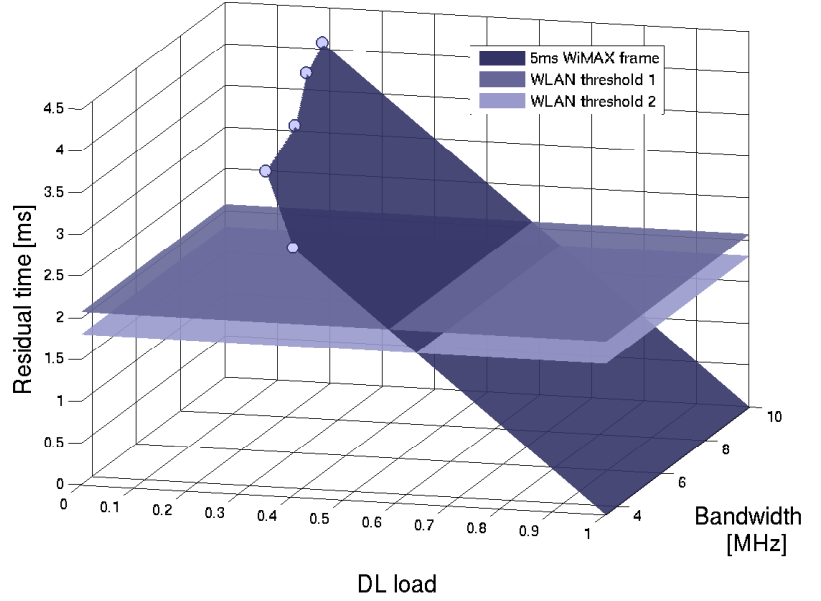

Fig. 5. Residual time with different levels of WiMAX DL load

TABLE III

MAXIMUM WiMAX DL LOAD (IN PERCENT OF FRAME SIZE)

\begin{tabular}{|c||ccccccc|}
\hline \multicolumn{1}{|c||}{$t_{\text {WLAN }_{\max }}$} & \multicolumn{7}{c|}{ Frame Duration [ms] } \\
& 2.5 & 4 & 5 & 8 & 10 & 12.5 & 20 \\
\hline \hline $1.806 \mathrm{~ms}$ & 27.8 & 54.9 & 63.9 & 77.4 & 81.9 & 85.6 & 91.0 \\
$2.074 \mathrm{~ms}$ & 17.0 & 48.2 & 58.5 & 74.1 & 79.3 & 83.4 & 89.6 \\
\hline
\end{tabular}

We define the maximum WiMAX DL load $L_{\max }$ as fraction of $T_{\text {frame }}$, under which the timing constraints of our solution still work:

$$
\mathrm{L}_{\max }=1-\frac{t_{\mathrm{WLAN}_{\max }}}{T_{\text {frame }}} .
$$

Table III gives the results for various WiMAX frame sizes and both WLAN thresholds. If $T_{\text {frame }}$ is far below the VoIP packetization interval, it is pretty likely that there is only one packet waiting in UL or DL. In this case, the smaller WLANthreshold applies. For larger $T_{\text {frame }}$ above $10 \mathrm{~ms}$, the second WLAN threshold is likely.

Overall, for 802.16e OFDMA with $5 \mathrm{~ms}$ frames, our solution is applicable if the DL part consumes not more than 63.9 percent of the frame duration (or $3.2 \mathrm{~ms}$ ).

Fig. 5 finally connects the results with and without other DL traffic graphically: the upper edge of the inclined plane represents the case with no other background traffic (and QPSK-1/2 MCS for all messages). Dependent on the WiMAX parameter combination, there is still space for other 18.1 percent $(3.5 \mathrm{MHz}$ bandwidth) and 39.9 percent $(10 \mathrm{MHz})$ of the WiMAX frame for the DL load.

\section{Vi. Conclusions And Future Research}

This paper presented a standard-compliant approach enabling ongoing WLAN communication as well as the WiMAX network entry using only a single, shared transceiver chain for both. Thereby, we alternate both technologies on small time scales. The evaluation of the novel scheme highlighted the timing constraints for WLAN and WiMAX. Our results identify the limits for different mobile profiles of the WiMAX forum as well as WLAN such that QoS constraints even of realtime traffic such as VoIP are not violated.

The results further motivate research regarding the support of real-time traffic over two RATs via single transceiver chains, thereby focusing on different reasons for the usage of alternative paths. In order to handle much different situations (e.g., regarding wireless channel conditions or bursty background traffic), both in WiMAX and WLAN, we will consider different policies for the prioritization of the timing between the two access technologies. For example, it may be important in other scenarios to give more channel access time to WLAN (i.e., prioritize the transport of VoIP data) and postpone subsequent steps of the WiMAX network entry process (in the valid range of the IEEE 802.16 standard) instead.

\section{ACKNOWLEDGMENTS}

This work has been partially supported by the projects EUCOAST (FP7-ICT- 248036), MEVICO (BMBF 01BU1014), as well as EU-TREND (FP7-ICT-257740).

\section{REFERENCES}

[1] X. Yan, Y. A. Sekercioglu, and S. Narayanan, "A survey of vertical handover decision algorithms in Fourth Generation heterogeneous wireless networks," Computer Networks, vol. 54, no. 11, pp. 1848 - 1863, 2010.

[2] M. Hollick, P. Mogre, C. Schott, and R. Steinmetz, "Slow and steady: Modelling and performance analysis of the network entry process in IEEE 802.16," in IEEE IWQoS 2007, jun. 2007, pp. $126-134$.

[3] U. Ramacher, "Software-defined radio prospects for multistandard mobile phones," Computer, vol. 40, pp. 62-69, 2007.

[4] Product Brief: PMB 2008, SMARTiTM WiMAX Single-chip dualband WiMAX / WLAN RF Transceiver IC with standard $I \& Q$ interface, Infineon Technologies AG. [Online]. Available: http://www.datasheetarchive.com/smarti*-datasheet.html

[5] Product Brief: Intel Centrino Wireless- $N+$ WiMAX 6150, Intel. [Online]. Available: http://www.intel.com/Assets/PDF/prodbrief/324748.pdf

[6] H.-H. Choi, O. Song, Y.-K. Park, and J.-R. Lee, "Performance evaluation of opportunistic vertical handover considering on-off characteristics of VoIP traffic," Vehicular Technology, IEEE Transactions on, vol. 59, no. 6, pp. 3115-3121, July 2010.

[7] M. Emmelmann, S. Wiethölter, and H.-T. Lim, "Opportunistic scanning: Interruption-free network topology discovery for wireless mesh networks," in IEEE WoWMoM, Kos, Greece, Jun. 2009, pp. 1 - 6.

[8] — "Influence of network load on the performance of opportunistic scanning," in IEEE LCN, Zurich, Switzerland, Oct. 2009, pp. 1-8.

[9] M. Emmelmann, S. Wiethölter, and H.-T. Lim, "Continuous network discovery using opportunistic scanning," European Patent PCT/EP 2010/003 774, pending.

[10] J. G. Atallah and M. Ismail, "Future 4G front-ends enabling smooth vertical handovers," Circuits and Devices Magazine, IEEE, vol. 22, no. 1, pp. $6-15,2006$.

[11] Nokia Siemens Networks, "Improving 4G coverage and capacity indoors and at hotspots with LTE femtocells," Whitepaper, 2011.

[12] WiMAX Forum, "Mobile System Profile Specification, Release 1.5, Common Part," WiMAX Forum, Tech. Rep. WMF-T23-001-R015v01, August 2009.

[13] S. Shin and H. Schulzrinne, "Measurement and analysis of the VoIP capacity in IEEE 802.11 WLAN," Mobile Computing, IEEE Transactions on, vol. 8, no. 9, pp. $1265-1279$, Sept. 2009.

[14] A. Markopoulou, F. Tobagi, and M. Karam, "Loss and delay measurements of Internet backbones," Comput. Commun., vol. 29, no. 10, pp. 1590-1604, June 2006.

[15] 802.16-2009: IEEE Standard for Local and metropolitan area networks, Part 16: Air Interface for Broadband Wireless Access Systems, IEEE Std., May 2009.

[16] IEEE 802.11, "Wireless LAN Medium Access Control (MAC) and Physical Layer (PHY) Specifications," IEEE Std 802.11-2007, June 2007. 